\title{
Complex anatomic variation in the brachial region
}

\author{
Th. Troupis, A. Michalinos, V. Protogerou, A. Mazarakis, P. Skandalakis \\ Department of Anatomy, Medical School, National and Kapodistrian University of Athens, Greece
}

[Received 10 July 2014; Accepted 4 August 2014]

\begin{abstract}
Authors describe a case of a complex anatomic variation discovered during dissection of the humeral region. On the right side, brachial artery followed a superficial course. Musculocutaneous nerve did not pierce coracobrachialis muscle but instead passed below the muscle before continuing in the forearm. On the left side, a communication between musculocutaneous and median nerve was dissected. Those variations are analytically presented with a brief review on their anatomic and clinical implications. Considerations on their embryological origin are attempted. (Folia Morphol 2015; 74, 1: 118-121)
\end{abstract}

Key words: superficial brachial artery, musculocutaneous nerve, median nerve, embryology

\section{INTRODUCTION}

Superficial brachial artery (SBA) is a brachial artery that courses above median nerve level [5]. It is a relatively rare anatomic variation with significant impact on a variety of therapeutic and surgical approaches including trauma cases, vascular access and endovascular therapy.

Terminal braches of brachial plexus are musculocutaneous nerve, median nerve radial nerve and ulnar nerve. Musculocutaneous nerve innervates muscles of front compartment of the humerus. It pierces coracobrachialis muscle and continues superficially as lateral cutaneous antebrachial nerve. Communications between musculocutaneous and median nerve are relatively common [1] with significant impact on muscle innervation and consequently on appearance of a variety of neuropathies, defects after trauma and alterations at surgical approaches. Musculocutaneous nerve not piercing coracobrachialis is a rare and less investigated variation with possible impact on coracobrachialis function [3].

In this study a case of coexistence of those variations is presented and analysed. Clinical impact is briefly reviewed and considerations on their developmental significance are attempted.

\section{CASE REPORT}

During dissection of the brachial region of a male cadaver at laboratory of Anatomy, National and Kapodistrian University of Athens, bilateral anatomic variations involving brachial plexus and brachial artery system were encountered. Primary dissection was made by students and further dissection by authors.

On the right side, brachial artery passed superficially to ansa medianis. At this level it gave a large branch that passed below ansa medianis and bifurcated into deep brachial artery and a well-developed collateral ulnar artery. SBA continued superficially to median nerve along its course and provided all typical branches. At elbow level it bifurcated into radial and ulnar artery. Also on the right side musculocutaneous nerve did not pierce coracobrachialis muscle but instead passed through a level between biceps brachii and coracobrachialis until lower third of the humerus where it continued as lateral antebrachial cutaneous nerve (Fig. 1). 


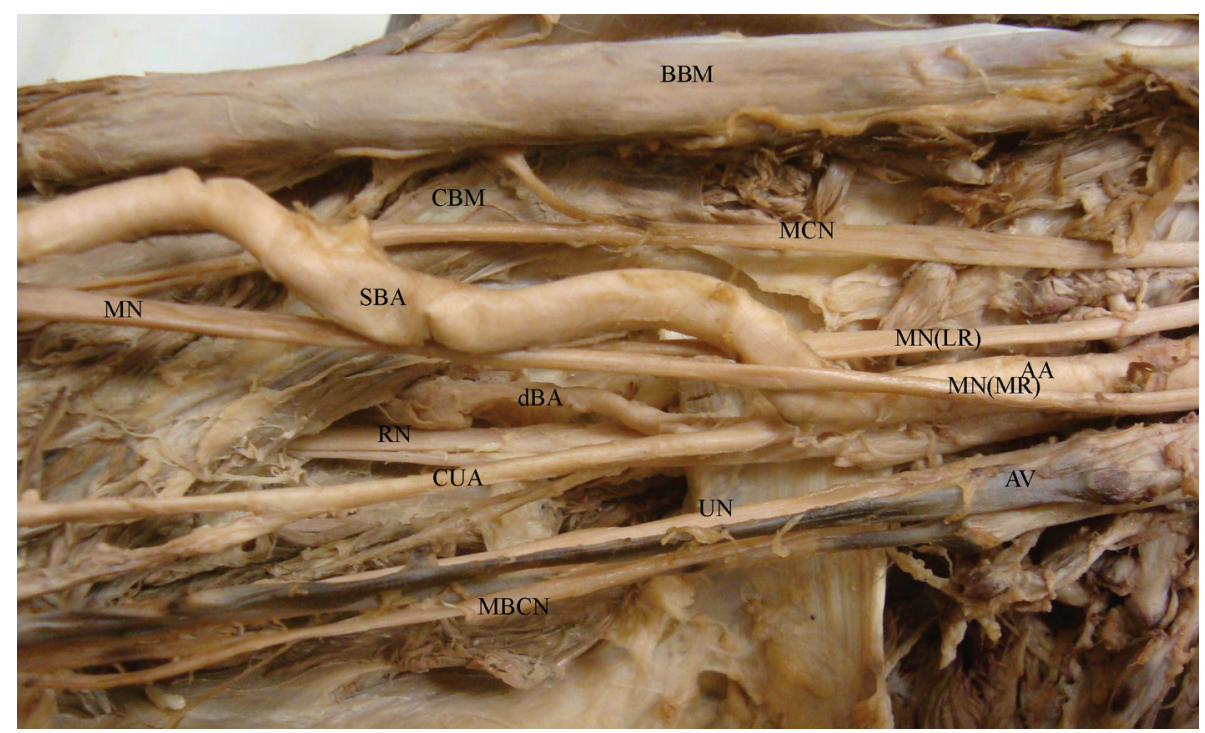

Figure 1. Right arm with superficial brachial artery and musculocutaneous nerve not piercing coracobrachialis muscle; BBM — biceps brachii muscle; $\mathrm{CBM}$ - coracobrachialis muscle; $\mathrm{MCN}$ - musculocutaneous nerve; MN — median nerve; SBA — superficial brachial artery; $\mathrm{MN}(\mathrm{LR})$ - median nerve, lateral root; $\mathrm{AA}$ — axillary artery; $\mathrm{MN}(\mathrm{MR})$ - median nerve, medial root; $\mathrm{dBA}$ — deep brachial artery; RN — radial nerve; CUA — collateral ulnar artery; UN — ulnar nerve; AV — axillary vein; MBCN — median brachial cutaneous nerve.

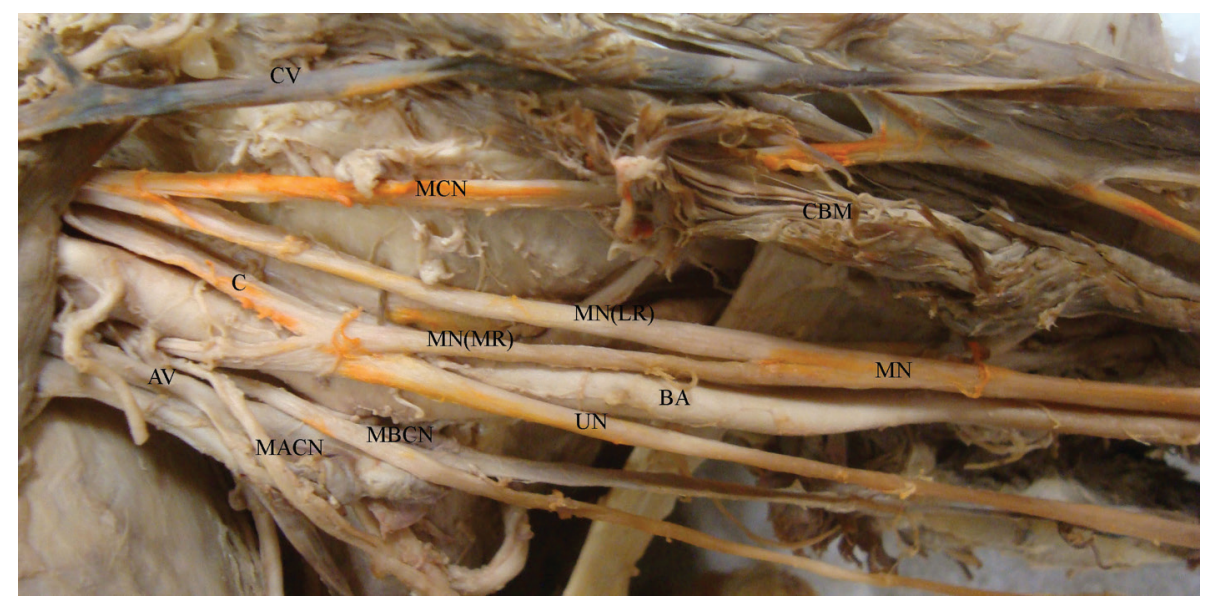

Figure 2. Left arm with communication between musculocutaneous and median nerve; BA — brachial artery; C — communication; CV — cephalic vein; MACN - median antebrachial cutaneous nerve; rest abbreviations as in Figure 1.

On left side, a communicating branch between musculocutaneous and median nerve existed before nerve's entrance at coracobrachialis muscle (Fig. 2).

No other variations concerning axillary, brachial and forearm region were encountered during dissection.

\section{DISCUSSION}

SBA is defined as brachial artery coursing above median nerve level. SBA can acquire its superficial course above or below ansa medianis [5]. SBA originating above ansa medianis is a relative rare variation with incidence $2-3.5 \%[5,8,9]$. It is commoner on the right side and in males [9]. SBA can be clinically important. During everyday practice it can be confused with a vein at elbow region leading to accidental drugs injection with possible toxicity, since liver is by-passed, or even limb necrosis [2]. Its significance for surgery is undoubted, especially in orthopaedics trauma cases. Vascular access for coronary by-pass or for arteriovenous fistula creation is affected since variant anatomy can lead to confusion during dissection or limited surgical intervention effectiveness. Finally, 
existence of SBA predisposes to various neuropathies due to median nerve compression [9].

Communications between musculocutaneous and median nerve are common, as high as 43\% [4]. Commonest description accounting for $70 \%$ of all musculocutaneous-median nerve communication is that of a branch originating from musculocutaneous to median nerve [1]. Its clinical significance is not clarified. Probably they disturb classical neurotome description with impact on movement and sensory defects in case of trauma or neuropathies [1]. They might increase dissection errors especially during emergency cases and are responsible of unexpected effects in some regional anaesthesia cases [7].

Musculocutaneous nerve not piercing coracobrachialis is a relatively rare and less investigated variation with incidence accounting between $3 \%$ and $10 \%$ across various studies [1, 3, 4]. el-Naggar [3] connected this rare variation with variations in coracobrachialis morphology: according to his study coracobrachialis consists of 2 heads, superficial and deep to musculocutaneous nerve with superficial head arising from tendon of biceps brachii and deep head from apex of coracoids process. When musculocutaneous nerve did not pierce coracobrachialis, the muscle consisted of only 2 head, showing that superficial head is the dominant one with main function stabilisation of tendon of biceps brachii during arm flexion. This comes in contrast with classical description of coracobrachialis, where origin of coracoids process is considered dominant. Another study [4] attributed protective function to this anatomic variation because coracobrachialis acts as anchorage to musculocutaneous nerve. Not piercing might mean more flexibility against trauma of flexion.

While typology of anatomic variations is well and analytically described and classified in terms of incidence and form, little is known about their aetiology. According to sprouting prototype, occurrence of superficial arteries is due to retention in vessels development or persistence of embryonic vessels. However modern studies do not completely confirm this theory. According to Rodriguez-Niedenfuhr et al. [8] development does not occur through sprouting but through capillary differentiation process in a proximal to distal fashion and in concordance with neural and skeletal limb elements development. Persistence, development or differentiation of capillaries that would normally regress gives rise in anatomic variations. It is also known that SBA and other superficial vessels are encountered in lower primitives [7].
Even less is known about brachial plexus morphogenesis. Embryologically, the brachial plexus appears as a single radicular cone of axons of spinal nerves, growing distally to reach the muscles and skin of the upper limb; later these axons divide to form ventral and dorsal divisions [10]. Communications between musculocutaneous and median nerve are usually interpreted as fibres that "escaped" lateral root of median nerve and re-insert through a separate communication [4]. Like superficial arm vessels, both communications between musculocutaneous and median nerve are known to occur in lower primitives, like monkeys or apes [1] while piercing of coracobrachialis is seen at chimpanzees but not gorillas or gibbons [3]. Variations in brachial plexus nerves might also be retention of a primitive pattern.

Variations of arterial and nerve system of upper limb are often studied separately while it is known that they often coexist and interfere. Miller [6] at his study in 1934 stated that those variations coexist at least at $8 \%$. According to him, in $41 \%$ of the anomalous cases the plexus is normal, whereas its relationship with the artery is not. Conversely, $14 \%$ of the cases show a normal artery in an abnormal relationship with the plexus. In $45 \%$ of the anomalies, the artery and plexus are mutually aberrant.

Aetiology of anatomic variations is not known. If it was purely genetic, all variations should be bilateral in a much higher percentage. If it was purely environmental, they should be chaotic and not amenable to any classification. Instead, especially in arm region, variations often coexist and are often similar to primitive patterns. This probably indicates that if typical anatomy is not possible due to defects or random events, final development occurs through choice between preserved patterns. Mediators of this process are not known but they might include oxygenation, haemodynamic or nutrition factors [8].

Anatomic variations have major clinical, surgical and embryological impact. Their existence can alter clinical images and modify results of surgical intervention. If unnoticed, they can be source of medical mistakes or causes of limited treatment effectiveness. While well-described, revisiting them is valuable and educative for clinicians because it helps maintaining interest and a high level of suspicion. Research on their embryology and origin has not reached final conclusions but they are probably connected to both genetic and environmental factors. It is possible they occur due to general disturbance to typical evolution prototype. 


\section{CONCLUSIONS}

Whether the origin of these anatomic variations, clinicians and, especially, surgeons should maintain a high level of suspicion for their existence and, if encountered, they should be ready to modify their therapeutic or surgical interventions so to ensure the best strategy for the patient's.

\section{REFERENCES}

1. Choi $D$, Rodríguez-Niedenführ $M$, Vázquez $T$, Parkin I, Sañudo JR (2002) Patterns of connections between the musculocutaneous and median nerves in the axilla and arm. Clin Anat NYN, 15: 11-17.

2. Claassen $H$, Schmitt $O$, Werner D, Schareck W, Kröger J-C, Wree A (2010) Superficial arm arteries revisited: Brother and sister with absent radial pulse. Ann Anat Anat Anz Organ Anat Ges, 192: 151-155.

3. el-Naggar MM (2001) A study on the morphology of the coracobrachialis muscle and its relationship with the musculocutaneous nerve. Folia Morphol, 60: 217-224.

4. Guerri-Guttenberg RA, Ingolotti M (2009) Classifying musculocutaneous nerve variations. Clin Anat NYN, 22: 671-683.

5. Lippert H, Pabst R (1985) Arterial variations in man. J. F. Bergmann, Munchen.

6. Miller R (1934) Observations upon the arrangement of the axillary artery and brachial plexus. Am J Anat, 64: 143-163.

7. Orebaugh SL, Williams BA (2009) Brachial plexus anatomy: normal and variant. ScienWorld J, 9: 300-312.

8. Rodríguez-Niedenführ M, Burton GJ, Deu J, Sañudo JR (2001) Development of the arterial pattern in the upper limb of staged human embryos: normal development and anatomic variations. J Anat, 199 (Part 4): 407-417.

9. Rodríguez-Niedenführ M, Vázquez T, Nearn L, Ferreira B, Parkin I, Sañudo JR (2001) Variations of the arterial pattern in the upper limb revisited: a morphological and statistical study, with a review of the literature. J Anat, 199 (Part 5): 547-566.

10. Saeed M, Rufai AA (2003) Median and musculocutaneous nerves: variant formation and distribution. Clin Anat NYN, 16: 453-457. 